\title{
A Robust Data-Driven Approach to the Decoding of Pyloric Neuron Activity
}

\author{
Filipa dos Santos ${ }^{1}$, P Andras ${ }^{2}$, DJ Collins ${ }^{3}$ and KP Lam ${ }^{4}$ \\ School of Computing and Mathematics \\ Keele University \\ Newcastle-under-Lyme, UK \\ Email: f.dos.santos@keele.ac.uk
}

\begin{abstract}
The combination of intra and extra-cellular recording of small neuronal circuits such as stomatogastric nervous systems of the crab (Cancer borealis) is well documented and routinely practised. Voltage sensitive dye imaging (VSDi) is a promising technology for the simultaneous monitoring of neuronal activities in such a system. However, integrating data obtained from optical VSDi and electrophysiological recording of the lateral ventricular nerve (lvn) is a complex and exacting task. Our early work demonstrated some of the concepts and principle involved. In this paper, we examine and report on the results obtained from the application of signal processing techniques to three datasets for which we had VSDi and Ivn data. Whilst significant challenges remain, we show that such an approach offers the possibility of real-time monitoring using automated analysis of VSDi data streams without the requirement for either extracellular (lvn) or intracellular recording.
\end{abstract}

Keywords- multiresolution signal processing, optical recording, singular spectrum analysis, stomatogastric ganglion, pyloric rhytm, voltage sensitive dye.

\section{INTRODUCTION}

The ability to locate circuit neurons and simultaneously record activity at single-cell resolution is fundamental to understanding the full scale network behaviour of neural systems. Studies of the stomatogastric nervous systems of the crab (Cancer borealis), for example, have afforded scientists numerous insights into the cellular and circuit mechanisms of rhythmic motor generators. Traditionally, methods developed to identify pattern generating neurons routinely require the laborious task of analysing the related neuron/network activities that are recorded intracellularly and extracellularly [1]-[4]. More importantly, many of these methods have relied on and been limited by the necessarily subjective judgement of the neuron location even in such a small nervous system, as the densely packed neurons that can exist in different layers are often covered by ganglion and nerve sheaths which impede access for recording electrodes and neuronal markers [5]. Voltage sensitive dye imaging (VSDi), an optical recording technique which can in principle report the membrane potential of multiple neurons, offers a promising alternative. Until recently, however, its use is largely confined to supplement intracellular recordings by enhancing the visibility of fine neural structures through staining and, to some extent, improving the signal-to-noise ratio in order to identify neurons in single-sweep recordings and synaptic events following the application of the so-called event/spike triggered averaging procedure [6]-[11].

From a signal processing viewpoint, our recent work has demonstrated the use of VSD as a sensor probe that optically represents the neural membrane oscillations, from which the pyloric rhythm (PR) of the stomatogastric ganglion (STG) of the crab might be accurately and reliably isolated, obviating the need for intracellular recordings. More significantly, the participating pyloric neurons (PY) have also been identified, using sophisticated signal processing procedures. The latter were developed to compare and correlate the optically recorded intensity of the fluorescence produced by the voltage-sensitive dye (Di-4-ANEPPS). The dye was bath-applied exogenously to the STG sample and the firing phases deconstructed from the activity patterns captured extracellularly through the lateral ventricular nerve (lvn) [12]-[13]. Specifically, the biologically meaningful and computationally well-defined duty cycle of the participating neurons was used as a biomarker to help identify the PY neuron/cell from the temporally captured sequence of images of the bath dyed STG sample [13].

This paper extends the above analysis of the pyloric constrictor neuron $(\mathrm{PY}, \mathrm{x} 5)$ to consider lateral pyloric (LP, $\mathrm{x} 1)$ and pyloric dilator (PD, $\mathrm{x} 2$ ) neurons based on the rhythmic and triphasic activity patterns routinely observable from the lvn recordings. The organization of this paper is as follows: Section II discusses the concepts and principles underlying our analysis from a signal processing perspective. In particular, it describes the well documented triphasic activity pattern present in the lvn. Section III describes the experimental setup from which the four datasets are collated and subsequently analysed in Section IV. In Section IV, the results are reported and discussed, and the major challenges that were addressed by this work are highlighted. Concluding remarks including future direction of work are presented in Section V.

\section{TRIPHASIC PATtern GenERATOR NEURONS}

Signals containing the pyloric rhythm are simultaneously captured from the bath dyed STG sample as image sequences and recorded as intensity fluroesence intensity alongside the electrophysiological recordings of the pyloric activities monitored on the lvn. The latter is principally comprised of the three key neuron types (PD, LP, PY) participating in the PR and, as such, provides a reference benchmark in terms of pyloric frequency, phase, well documented triphasic

Copyright: 978-1-5386-0446-5/17/\$31.00 C2017 IEEE 
relationships and most relevantly, the respective duty cycles of the participating neurons. Fig. 1 summarises the above, with examples included to help illustrate the sampled signals (blue) typically present in both the bath dyed STG and the lvn.

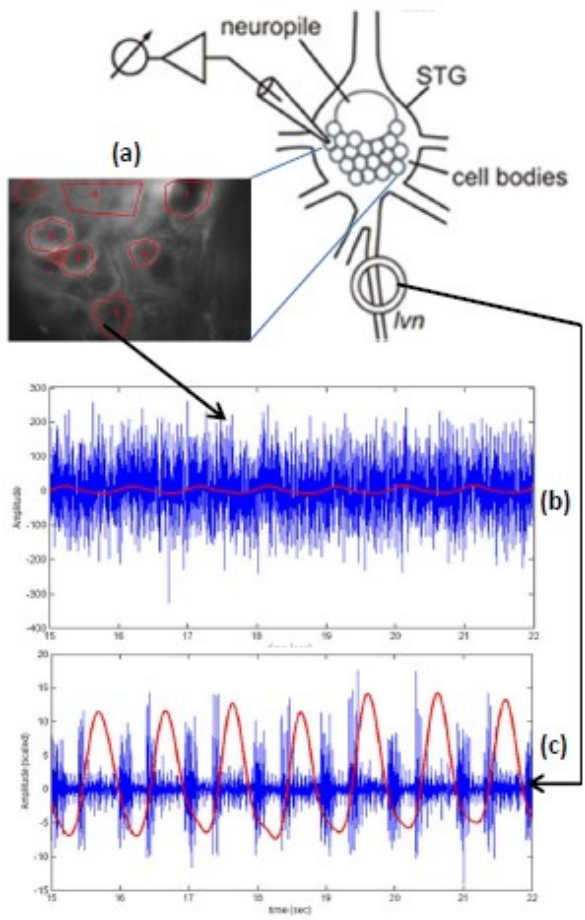

Fig. 1. Schematic diagam of the STG system. The dye was applied to the STG sample using a pipette. (a) A high resolution picture of the crab STG, with regions of interest of each cell marked for identification. (b) A typical signal (with SNR at $\sim 20 \mathrm{~dB}$ ) captured as intensity of the fluorence produced by the VSD over time (blue), with the isolated PR (red) being displayed on the top of it. (c) The same PR (red) is shifted/delayed to match with the identified (PY) phase of each of the plyoric cycle recorded on the lvn (blue), which has been scaled to facilitate visual examination.

A key objective of the experiment is to compare and map the STG neuron activity (the PR) optically recorded (as changes in fluorescence over time) in the dye bath containing the STG sample with the extracellularly captured pyloric motor pattern on the lvn, thus allowing the neuron/cell type for the individual region of interest (ROI) shown in Fig. 1(a) to be identified . Here, it should be noted that:

1. The relatively low yield of the potential-dependent fluorescence change $(2-10 \% / 100 \mathrm{mV})$ achieved by the Di-4-ANEPPS dye used in our study make the PR extraction from the recorded dye bath/images a complex signal processing task [12]. See Fig. 1(b).

2. The inherently triphasic PR monitored on the lvn by the activity of three distinct types of neurons; namely, lateral pyloric (LP, one cell) neuron, pyloric constrictor (PY, 5 cells) neurons and pyloric dilator (PD, two cells) neurons, is recorded as extracellular neuronal potentials (i.e. spikes) corresponding to the highly coordinated oscillatory activities of the participating neurons. As with most extracellular electrode recordings of neuronal activity, such recording is typically displayed in the form of a high frequency sequence or train of spikes (i.e. bursts). See Fig. 1(c).

3. The timing lag between the optical and lvn recordings of the characteristic spike peaks for the participating neurons due to the axonal transmission delay between the ganglion and the extracellular recording site is unknown and dependent upon (i) the biological/STG sample, (ii) the equipment ensemble and (iii) the experiment setup. In the example given in Fig. 1(c), it is apparent that both the amplitude and duty cycle of the PR varies quite considerably over successive pyloric cycles.

\section{EXPERIMENTAL SETUP}

\section{A. STG sample and dye (Di-4-ANEPPS) preparation}

Adult Cancer pagurus were obtained from Hodgkinson Fresh Fish, Manchester; they were kept in artificial seawater tanks (red sea salt, Red Sea) at $12-14^{\circ} \mathrm{C}$ with $12 \mathrm{~h}$ light-dark cycle. The STG sample was prepared following the protocol described in [14] and was stained with Di-4-ANEPPS dye (Cambridge Bioscience, UK) and subsequently washed out using a saline flow.

Di-4-ANEPPS (Cambridge Bioscience, Cambridge, UK) used by bath application on the STG was surrounded by a petroleum jelly-well. The dye was kept in stock solution $5 \mathrm{mg}$ di-4-ANEPPS dissolved in $1 \mathrm{ml}$ Pluronic F-127 $(20 \%$ solution in DMSO). On the day of each experiment, $10 \mu \mathrm{l}$ of the stock solution was mixed with $500 \mu$ l of saline. The dyed prep was then left in the fridge for approximately 20 minutes. This done, the petroleum jelly well from around the STG was removed, and the dye was washed out using a saline flow.

\section{B. Extracellular and image recording}

The extracellular recording was carried out on the lvn, with the differential signal filtered and amplified by an AC differential amplifier before it was converted using Spike2 v8.07 (Power 1401, Cambridge Electronic Design, UK). Using the MiCAM02 imaging system (SciMedia Ltd., Japan), the image of the dye-bath containing the stained STG sample was also recorded simultaneously with the lvn recording. Here, a high-resolution (192x128) image was first taken for each experiment to help (better) identify the individual cells within the field of view of the microscope from a series of low resolution $(48 \times 32)$ co-registered images recorded over the course of a recording; see further description in Data Collection below. These low-resolution images form a (3-D) image stack which, for the purpose of our studies, provides a series of 21,840 images sampled over the course of a recording, offering a temporal resolution of $1.5 \mathrm{~ms}$. The biological sample/prep was seated on a standard upright fluorescence microscope (BX51WI, Olympus Corporation, Tokyo, Japan) which is equipped with a 20x mounted objective. The lighting was delivered from a $150 \mathrm{~W}$ halogen light source (HL-151, Moritex Corporation, Tokyo, Japan) with a computer controlled shutter which facilitates 
adjustment of the light levels to minimise potential phototoxic damage. A wide green excitation filter was also used (480550 nm, MSWG2, Olympus Corp., Tokyo, Japan) in accordance with the VSD specifications.

\section{Data collection and preprocessing}

The data obtained from the optical recordings described in $A$. above was extracted using the BVAna imaging software (SciMedia Ltd., Tokyo, Japan), which exports the data from the 3-D image stack stored for each recording into CSV formatted spreadsheets.

Here, a number of pixels were meticulously chosen from each identifiable cell, collated as the distinct ROI, within the fieldof-view of the high resolution (2-D) image described above; see Fig. 1(a). These pixels were then mapped to the corresponding pixels in the (low-resolution) image stack, from which values of the selected pixels were extracted in the form of 1-D time/signal sequences from successive images of the 3-D stack; see Fig. 1(b) for illustration. This process is repeated for areas covering all other cells, or ROIs ${ }^{1}$, that have been identified, including importantly the neuropil.

\section{SIGNAL PROCESSING AND ANALYSIS}

The optically recorded data from the bath dyed STG sample is processed separately from the extracellular recording of triphase pyloric activity/pattern monitored on the lvn. First, the multiresolution procedure described in [12] was applied to the individually demarcated ROIs in order for the relevant signal sub/components of the PR, if present, to be accurately extracted. Further, the signal components bearing the first and second harmomics of the pyloric frequency must also be isolated, to enable estimation of the duty cycle and thus identification of the participating pyloric neurons [13]. Second, for the extracellularly captured data on the lvn, the primary task is to separate the individual phase of the PR from each computed pyloric cycle, in accordance with the spiking/activity pattern characteristic of the respective participating neuron. Here, the spontaneous and characteristic motor pattern consisting of the highly synchronised activity of the PD-timed, LP-timed or PY-timed neurons were analysed and segmented using a conventional wavelet-based multiresolution procedure which enable the deconstruction of regular and predictable phasing of the pyloric activities.

\section{A. PR isolation from ROIs}

A key challenge in isolating the pyloric rhythm from each collated ROI is the relatively poor SNRs achieved by the VSDs. Specifically, in addition to uncorrelated white noise that covered almost the entire frequency spectrum $(0$ to $333 \mathrm{~Hz})$, the original/raw signal contains highly correlated (sinusoidal) signal components as well as bandlimited noise process which both seemed unrelated to the PR. To address this, the spectral estimation technique of Singular Spectrum Analysis (SSA)

1 It is noted that the number of pixels extracted from each cell varies, depending on the size of the visibly identifiable area of the neuron. Further, by estimating the duty cycle through extracting the first and second harmonics of the pyloric frequency, we can identify the PR generating neuron [13]. which is generally known to provide good resolution and frequency estimation characteristics was chosen to identify and (subsequently) extract the PR [12,16]. Here, the SSA was developed as a signal decomposition procedure that separates the original signal into two subspaces: (i) a signal consisting of the mixture of a small number of independent and interpretable components, and (ii) a structureless noise. More specifically, a multiresolution algorithm similar to the wavelet analysis of signals was constructed using the so-called sequential SSA (sSSA). The basic SSA is applied repeatedly with different parameters to the residuals from the previous analysis in order to resolve the mixing problem of signal components from each ROI within the collated time sequence. The latter has been shown to display a complex form [12].

For each STG sample/experiment, the multiresolution procedure of the s-SSA described above was first applied to the neuropil that was known and demonstrated to contain signals which are domoniated by the pyloric rhythm/frequency in the corresponding STG sample. In addition to providing a reference for comparision with the extracted signal/components from each cell/ROI, this also served to determine the parameters used with the s-SSA when it was subsequently applied to each identified ROI, allowing the estimation of the signal-to-noise ratio (SNR), the ratio of harmonics $\left(\mathrm{RH}_{\mathrm{xy}}\right)$ and the corresponding duty cycle (DC) computed for each candidate neuron/cell.

\section{B. Pyloric phase separation on the lvn}

The deconstruction of the highly structured and regular phasing of the pyloric activities recorded on the lvn is constructed using the widely studied multi-resolution technique of time-frequency analysis [13,15]. Importantly, this was assisted by the s-SSA procedure described above, which sought to remove noise from the lvn whilst selectively preserving the pyloric frequency and its higher order harmonics. Here, the analytic Morlet wavelet, known for its superior time localisation, was used to better localise the transients between individual phases of the pyloric rhythm from the (now) relatively noise-free recording on the lvn. This was achieved by selecting a frequency band in the constructed spectrogram where the amplitude response produced in the PY-timed pyloric cycle and the quiescent phase/period are significantly lower $(\sim 75 \%)$ than that in either of the LP-timed and PD-timed pyloric cycle. Using a simple thresholding procedure, the respective time periods of PY phase were segmented from the individual pyloric cycle recorded on the lvn and, subsequently, the corresponding duty cycle of the PY phase was computed [13].

In this study, the procedure described above has been extended to enable estimation of the respective duty cycles of the PD and LP phases. In summary, this was achieved by noting that the highly structured and predicable phasing of the PR would allow the start and end point of the triphasic patterns led by the indiviudal participating neurons (PD, LP and PY) to be computed easily following the successful segmentation of the PY and quiescent phases of the PR monitored on the lvn. The procedure is illustrated in Fig. 2. 


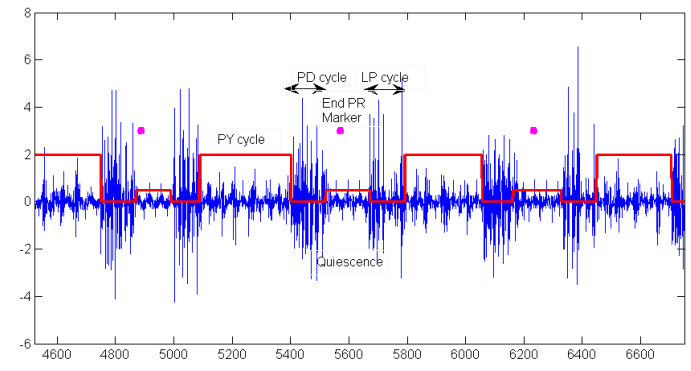

Fig. 2. Reconstruction of the PY burst train (red) with successive quiesent phases/periods (red) on the lvn recording (blue). An example of the the PD and LP bursts are shown with reference to the sucessive PY pulses and the quiesent phases of each PR cycle. To aid visual examination, the end-of-cycle marker (magenta) are also included to mark the end of each of the PR cycle which coincides with the end of the corresponding PD burst.

\section{RESULTS AND DisCUSSIONS}

We selected three datasets from over 40 experimental studies. Sets were chosen that covered a wide range of pyloric frequencies and a spread of SNR within the dataset. The results are displayed in Tables 1 to 3 . In all tables, the columns are as follows: $\mathrm{ROI}=$ Cell/ROI identifier, \#NP = Number of pixels in the ROI, $\mathrm{f}_{1}=$ Main/peak frequency detected $(\mathrm{Hz}), \mathrm{f}_{2}=$ Second main/peak frequency, SNR = Estimated Signal to Noise Ratio (dB), $R_{21}=$ Ratio of the second harmonic to the first, $D_{21}=$ the corresponding duty cycle $(\mathrm{x} / 100)$. In each of these tables, the extracted PR from the optical recording as well as the computed duty cycle (DC) of the (three) respective participating neurons (PD, LP and PY) monitored on the lvn are given in the table header/title as reference.

TABLE I. $\quad \mathrm{PR}=0.7324 \mathrm{~Hz}: \mathrm{DC}(\mathrm{PD})=0.123, \mathrm{DC}(\mathrm{LP})=0.328, \mathrm{DC}(\mathrm{PY})=0.384$

\begin{tabular}{|c|c|c|l|l|l|l|}
\hline ROI & \#NP & \multicolumn{1}{|c|}{$\mathbf{f}_{\mathbf{1}}$} & \multicolumn{1}{|c|}{$\mathbf{f}_{\mathbf{2}}$} & \multicolumn{1}{|c|}{$\mathbf{S N R}$} & $\mathbf{R}_{\mathbf{2 1}}\left(\mathbf{D C}_{\mathbf{2 1}}\right)$ & $\mathbf{R}_{\mathbf{3 1}}\left(\mathbf{D C}_{\mathbf{3 1}}\right)$ \\
\hline 1 & 18 & 0.8748 & 0.7324 & -31.012 & $1.91(0.1)$ & $4.34(0.1)$ \\
\hline 2 & 44 & 0.7528 & 0.7324 & -18.19 & $0.46(0.26)$ & $0.008(0.36)$ \\
\hline 3 & 14 & 0.7528 & 0.7324 & -19.14 & $0.42(0.28)$ & $0.062(0.42)$ \\
\hline 4 & 16 & 0.8748 & $\mathrm{n} / \mathrm{a}$ & -27.78 & $0.0014(0.49)$ & $0.99(0.1)$ \\
\hline 5 & 37 & 0.7324 & $\mathrm{n} / \mathrm{a}$ & -12.29 & $0.054(0.43)$ & $0.14(0.24)$ \\
\hline 6 & 29 & 0.7324 & 0.7 & -22.05 & $0.019(0.46)$ & $0.62(0.13)$ \\
\hline 7 & 33 & 0.7528 & 0.7324 & -20.03 & $0.14(0.38)$ & $0.196(0.22)$ \\
\hline 8 & 16 & 0.7324 & 0.8748 & -22.87 & $0.114(0.39)$ & $0.42(0.17)$ \\
\hline 9 & 20 & 0.8952 & 0.7324 & -24.97 & $0.385(0.29)$ & $0.24(0.21)$ \\
\hline 10 & 17 & 0.7324 & $\mathrm{n} / \mathrm{a}$ & -9.41 & $0.019(0.46)$ & $0.04(0.40)$ \\
\hline 11 & 23 & 0.8952 & $\mathrm{n} / \mathrm{a}$ & -26.35 & $1.07(0.1)$ & $0.60(0.14)$ \\
\hline 12 & 33 & 0.8956 & $\mathrm{n} / \mathrm{a}$ & -29.7 & $1.75(0.1)$ & $2.12(0.1)$ \\
\hline 13 & 19 & 0.7324 & $\mathrm{n} / \mathrm{a}$ & -14.73 & $0.11(0.39)$ & $0.056(0.41)$ \\
\hline 14 & 24 & 0.7324 & $\mathrm{n} / \mathrm{a}$ & -18.99 & $0.054(0.43)$ & $0.147(0.24)$ \\
\hline 15 & 32 & 0.7528 & 0.7324 & -12.24 & $0.187(0.36)$ & $0.15(0.24)$ \\
\hline 16 & 12 & 0.8952 & $\mathrm{n} / \mathrm{a}$ & -24.08 & $0.067(0.42)$ & $0.16(0.23)$ \\
\hline
\end{tabular}

In each of the tables, we also highlight the regions that display the pyloric frequency as either the first or second major frequency in the power spectrum. The basis for determining the pyloric frequency was described earlier in section $4 \mathrm{~A}$. In most cases, the SNR played an important part in determining our ability to locate the pyloric rhythm. Where signal to noise ratio is high (better than $-20 \mathrm{~dB}$ ), the pyloric frequency is readily detectable within the power spectrum: it appears as a sharp peak. In Table 1 , this is particularly clear. The opposite also applies - when SNR is low the peak detection poor and often erroneous.

When the pyloric frequency was detected we sought to establish whether the neuron responsible was in the pyloric circuit (consisting of $1 \times 2 P, 2 x P D$ and $5 x P Y$ ). In each of the tables we extracted the respective duty cycle of the neuron by calculating the R21 and R31 ratios and hence the corresponding duty cycle. This was compared with the duty cycle calculated from the recording on the lvn in the time rather than the frequency domain.

TABLE II. $\quad \mathrm{PR}=0.8748 \mathrm{~Hz}: \mathrm{DC}(\mathrm{PD})=0.128, \mathrm{DC}(\mathrm{LP})=0.156, \mathrm{DC}(\mathrm{PY})=0.347$

\begin{tabular}{|c|c|c|l|l|l|l|}
\hline ROI & \#NP & \multicolumn{1}{|c|}{$\mathbf{f}_{\mathbf{1}}$} & \multicolumn{1}{|c|}{$\mathbf{f}_{\mathbf{2}}$} & \multicolumn{1}{|c|}{$\mathbf{S N R}$} & \multicolumn{1}{|c|}{$\mathbf{R}_{\mathbf{2 1}}\left(\mathbf{D C}_{\mathbf{2 1}}\right)$} & \multicolumn{1}{|c|}{$\mathbf{R}_{\mathbf{3 1}}\left(\mathbf{D C}_{\mathbf{3 1}}\right)$} \\
\hline 1 & 16 & 0.8748 & 0.6917 & -28.99 & $0.0377(0.44)$ & $0.07(0.43)$ \\
\hline 2 & 12 & 0.7121 & $\mathrm{n} / \mathrm{a}$ & -37.61 & $1.25(0.1)$ & $0.17(0.23)$ \\
\hline 3 & 12 & 0.8454 & $\mathrm{n} / \mathrm{a}$ & -32.95 & $0.032(0.44)$ & $0.30(0.20)$ \\
\hline 4 & 18 & 0.8748 & $\mathrm{n} / \mathrm{a}$ & -34.32 & $0.45(0.27)$ & $0.30(0.20)$ \\
\hline 5 & 16 & 0.9359 & $\mathrm{n} / \mathrm{a}$ & -43.61 & $1.06(0.1)$ & $1.66(0.1)$ \\
\hline 6 & 32 & 0.6917 & n/a & -36.08 & $0.731(0.17)$ & $0.25(0.21)$ \\
\hline 7 & 19 & 0.6714 & n/a & -37.04 & $0.044(0.43)$ & $0.098(0.46)$ \\
\hline 8 & 20 & 0.7121 & $\mathrm{n} / \mathrm{a}$ & -35.65 & $0.39(0.28)$ & $0.73(0.11)$ \\
\hline 9 & 22 & 0.8545 & n/a & -31.57 & $0.035(0.44)$ & $0.18(0.23)$ \\
\hline 10 & 19 & 0.6917 & n/a & -35.97 & $0.009(0.47)$ & $0.066(0.42)$ \\
\hline 11 & 20 & 0.6917 & 0.8748 & -32.37 & $0.389(0.29)$ & $0.24(0.21)$ \\
\hline 12 & 16 & 0.6917 & n/a & -37.03 & $0.599(0.21)$ & $0.69(0.12)$ \\
\hline 13 & 24 & 0.8545 & n/a & -32.164 & $0.346(0.3)$ & $0.045(0.40)$ \\
\hline 14 & 21 & 0.7121 & n/a & -43.32 & $4.31(0.1)$ & $0.411(0.17)$ \\
\hline 15 & 26 & 0.8545 & n/a & -39.17 & $2.11(0.1)$ & $0.55(0.14)$ \\
\hline 16 & 15 & 0.6914 & n/a & -37.4 & $1.62(0.1)$ & $0.0078(0.31)$ \\
\hline 17 & 17 & 0.7121 & n/a & -51.14 & $4.84(0.1)$ & $9.16(0.1)$ \\
\hline
\end{tabular}

It can be seen that, in most cases, the duty cycles estimated by computing $\mathrm{R}_{21}$ and $\mathrm{R}_{31}$ in the frequency domain differ (bearing most similarity with the PY neurons). However, we consider $R_{21}$ to be the more reliable ratio as the second harmonic is generally much larger in amplitude than the third harmonic in the spectra obtained and, therefore, less prone to noise. More importantly, it should also be noted that these ratios derived from the reference DCs of the pyloric neurons (i.e. PD, LP and PY) are modeled previously on the lvn as a rectangular train pulse in the time domain [13]. Thus the parameters used for the s-SSA procedure might have to be fine- 
tuned to further extract from the relevant harmonic(s) of the pyloric frequency from each of the potential candidate/cell highlighted in the tables above, in order that the participating pyloric neurons could be identified (in the time domain). For example, this can be illustrated by candidate cell 13 in Table I, where the reference DC of the PY cell was estimated (from the lvn recording) to be 0.384 . Here, we adopted the phasematching technique which we have developed previously in [13] to estimate the time delay between the optical and lvn recording using the Hilbert transformation approach. In essence, the instantaneous phases of the PY-cycle separate from the individual recordings obtained from the respective Hilbert spectra were compared in order to find the best match [17]-[18]. This is illustrated in Fig. 3, where it is shown that the timing lag between the individual PY cell and the lvn maintains a fairly constant mean phase shift of 2.47 radians, with s.t.d $=0.45 \mathrm{rad}$.

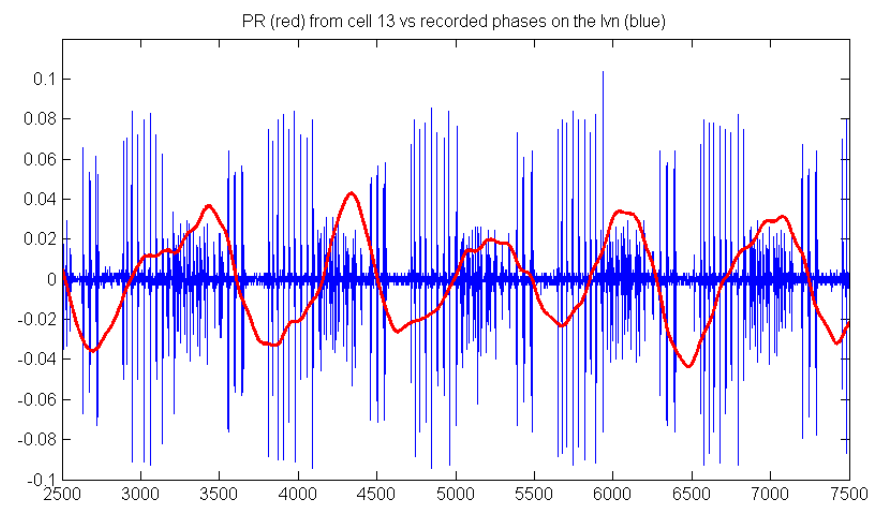

Fig. 3. The extracted PR (red) from cell 13 (Table I) is shifted/delayed to match with the identified (PY) phase of the plyoric activities recorded on the lvn (blue). The close alignment of the two signals is evident.

TABLE III. $\quad \mathrm{PR}=1.282 \mathrm{~Hz}: \mathrm{DC}(\mathrm{PD})=0.182, \mathrm{DC}(\mathrm{LP})=0.188, \mathrm{DC}(\mathrm{PY})=0.268$

\begin{tabular}{|c|c|c|l|l|l|l|}
\hline ROI & \#NP & $\mathbf{f}_{\mathbf{1}}$ & $\mathbf{f}_{\mathbf{2}}$ & $\mathbf{S N R}$ & $\mathbf{R}_{\mathbf{2 1}}\left(\mathbf{D C}_{\mathbf{2 1}}\right)$ & $\mathbf{R}_{\mathbf{3 1}}\left(\mathbf{D C}_{\mathbf{3 1}}\right)$ \\
\hline 1 & 15 & 1.404 & 1.282 & -23.25 & $0.056(0.42)$ & $0.02(0.30)$ \\
\hline 2 & 14 & 1.282 & 1.424 & -19.97 & $0.35(0.30)$ & $0.06(0.27)$ \\
\hline 3 & 14 & 1.404 & $\mathrm{n} / \mathrm{a}$ & -31.79 & $3.70(0.1)$ & $1.86(0.1)$ \\
\hline 4 & 11 & 1.404 & $\mathrm{n} / \mathrm{a}$ & -28.83 & $0.822(0.14)$ & $0.19(0.23)$ \\
\hline 5 & 14 & 1.282 & $\mathrm{n} / \mathrm{a}$ & -11.44 & $0.128(0.38)$ & $0.05(0.40)$ \\
\hline 6 & 14 & 1.282 & 1.404 & -19.68 & $0.126(0.39)$ & $0.15(0.24)$ \\
\hline 7 & 16 & 1.424 & 1.282 & -24.52 & $0.078(0.41)$ & $0.07(0.42)$ \\
\hline 8 & 23 & 1.404 & $\mathrm{n} / \mathrm{a}$ & -32.14 & $0.456(0.26)$ & $1.61(0.1)$ \\
\hline 9 & 20 & 1.404 & $\mathrm{n} / \mathrm{a}$ & -34.43 & $0.57(0.23)$ & $0.65(0.13)$ \\
\hline 10 & 9 & 1.424 & $\mathrm{n} / \mathrm{a}$ & -35.83 & $0.68(0.19)$ & $0.20(0.23)$ \\
\hline 11 & 13 & 1.282 & $\mathrm{n} / \mathrm{a}$ & -16.24 & $0.12(0.39)$ & $0.01(0.32)$ \\
\hline 12 & 13 & 1.282 & $\mathrm{n} / \mathrm{a}$ & -12.18 & $0.25(0.33)$ & $0.01(0.31)$ \\
\hline 13 & 13 & 1.282 & $\mathrm{n} / \mathrm{a}$ & -12.08 & $0.12(0.38)$ & $0.01(0.29)$ \\
\hline 14 & 15 & 1.404 & $\mathrm{n} / \mathrm{a}$ & -31.42 & $0.21(0.35)$ & $0.50(0.16)$ \\
\hline 15 & 16 & 1.383 & $\mathrm{n} / \mathrm{a}$ & -24.89 & $0.10(0.40)$ & $0.21(0.22)$ \\
\hline
\end{tabular}

It should be noted that, in passing, the decoding of the lvn recording in order to define accurately the triphasic relationship of the participating neurons (PD, LP and PY) in the PR generator circuit [13] and, importantly, their subsequent mapping or matching to the PR extracted from the VSDi data obtained from the bath dyed STG sample presented some of the most challenging signal processing tasks for our studies [18]. Here, the Hilbert transformation approach was demonstrated to provide both an accurate and effective means to estimate the practically unknown phase shift between the optical recording of STG sample from the dye bath and the extracellular recording monitored on the lvn [18]. Generally speaking, the $\mathrm{PY}$ is the longest duty cycle and therefore the easiest to distinguish by means of the ratio. Interestingly, however, we note that, counter to general belief, the relationship between the LP and PD duty cycles can be variable as is evidenced between Tables I and III, plus Tables II and III.

At present, the main limitation of this methodology is the fact that the duty cycle on its own is not, as yet, sufficiently discernible to automatically determine the neuronal type in the pyloric circuit. This is particularly the case given the relatively similar and comparatively narrow duty cycle widths of the LPled and PD-led phases/cycles that were estimated over the full length of lvn recording. Moreover, processing of the lvn recording by itself could be very challenging, often requiring some minor manual adjustments because of naturally occurring variability in the bursting cycle of the participating neurons in the pyloric circuit. This may be a serious obstacle to automatic real-time analysis. Finally, we are also aware of considerable variability in the signal to noise ratio across experiments. Specifically, the noticeably poorer SNR demonstrated in Table II is evident. Closer examinations of the power spectra obtained across the dataset reveals that much of noise/power was localized in the very low end of the frequency spectrum (well) below the computed PR (which is also validated by the accompanying lvn recording). However, this could have a range of explanations which we need to understand with a view to obtaining greater consistency and hence improved performance.

\section{CONCLUSION AND FUTURE WORK}

We have shown that advanced signal processing techniques have considerable potential for discerning the participating neurons in the pyloric circuit of Cancer pagurus using VSDi. Using duty cycles as a means of identification of the neuronal types can be reliable particularly when the SNR is favorable or sufficiently high. As such, this offers a real possibility of realtime automated monitoring of cell systems.

At present, variability in the duty cycles is not fully understood and signal to noise ratios are both variable and poor. We believe that these problems can be overcome with improvement in experimental technique coupled with, for example, high speed and higher resolution imaging equipment that is already available commercially. Furthermore, using previously developed techniques for spectral/component analysis [19]-[20], we also believe it will be possible to monitor neuronal systems without the requirement for the challenging task of lvn recording, by producing, for example, 
spectral reference signatures for the specific neuronal system under study.

\section{REFERENCES}

[1] M. P. Nusbaum and M. P. Beenhakker, "A small-systems approach to motor pattern generation," Nature, vol. 417, pp. 343-350, May 2002.

[2] E. Marder and D. Bucher, "Understanding circuit dynamics using the stomatogastric nervous system of lobsters and crabs," Annu. Rev. Physiol., vol. 69, pp. 13.1-13.26, September 2006.

[3] U. B. S. Hedrich, F. Diehl, and W. Stein, "Gastric and pyloric motor pattern control by a modulatory projection neuron in the intact crab Cancer pagurus," J. Neurophysiol., vol. 105, pp. 1671-1680, February 2011.

[4] C. Derby and M. Thiel, Nervous Systems and Control of Behavior (The Natural History of Crustacea), Volume 3. New York: Oxford University Press, 2014.

[5] CJ Goldsmitm, C Städele, and W Stein, Optical Imaging of Neuronal Activity and Visualization of Fine Neural Structures in Non-Desheathed Nervous Systems, PLoS One. 9(7): e103459, 2014.

[6] E. S. Hill, A. M. Bruno, and W. N. Frost, "Recent developments in VSD imaging of small neuronal networks," Learn. Mem., vol. 21, pp. 499-505, September 2014.

[7] V. Tsytsarev, et al., "Recent progress in voltage-sensitive dye imaging for neuroscience," J. Nanosci. Nanotechnol., vol. 14, no. 7, pp. 4733-4744, July 2014.

[8] W. Stein and P. Andras, "Light-induced effects of a fluorescent voltage-sensitive dye on neuronal activity in the crab Stomatogastric Ganglion," J. Neurosci. Methods, vol. 188, pp. 290-294, March 2010.
[9] W. Stein, C. Städele, and P. Andras, "Single-sweep voltagesensitive dye imaging of interacting identified neurons," J. Neurosci. Methods, vol. 194, pp. 224-234, October 2011.

[10] C. Städele, P. Andras, and W. Stein, "Simultaneous measurement of membrane potential changes in multiple pattern generating neurons using voltage sensitive dye imaging," J. Neurosci. Methods, vol. 203, pp. 78-88, September 2012.

[11] S. Preuss and W. Stein, "Comparison of two voltage-sensitive dyes and their suitability for long-term imaging of neuronal activity," PLoS One, vol. 8, no. 10, pp. 1-13, October 2013.

[12] F. Dos Santos, P. Andras \& K.P. Lam:, A Multi-resolution Approach to the Extraction of the Pyloric Rhythm, to appear in Procs IEEE TSP 2017.

[13] F. Dos Santos, P. Andras \& K.P. Lam:, Towards an Accurate Identification of Pyloric Neuron Activity with VSDi, accepted for publication in ICANN 2017, Italy, September 2017.

[14]G. J. Gutierrez and R. G. Grashow, "Cancer borealis Stomatogastric Nervous System dissection," J. Vis. Exp., pp. 15, January 2009.

[15] Akansu, AN \& Haddad RA.: Multiresolution Signal Decomposition (2nd Ed) Transforms, Subbands, and Wavelets, AP, 2000.

[16] N. Golyandina and A. Zhigljavsky, Singular Spectrum Analysis for Time Series. New York: Springer, 2013.

[17] Hahn, S.: Hilbert transforms in signal processing, Artech House, Inc., Norwood, 1996.

[18] F. Dos Santos, K.P. Lam \& P. Andras:, Neuron classification in the Stomatogastric ganglion using voltage-sensitive dye imaging and signal processing tools (POSTER), 26 $6^{\text {th }}$ Annual Computational Neural Science Meeting, Belgium, August 2017.

[19] K. Lam, J. C. Austin, and C. R. Day, “A coarse-grained spectral signature generator," 2007 , p. $63560 \mathrm{~S}$.

[20] K. Lam and R. Emery, "Image pixel guided tours: a software platform for non-destructive x-ray imaging," 2009, p. $72450 \mathrm{~N}$. 